\title{
August 2016 Critical Care Case of the Month
}

\section{Jillian L. Deangelis, APRN, CNP \\ Theodore Loftsgard APRN, ACNP}

\author{
Department of Anesthesiology \\ Mayo Clinic Minnesota \\ Rochester, MN USA
}

\section{History of Present IIIness}

The patient is a previously healthy, albeit anxious, 15-year-old girl seen by her primary care physician. She has had several months of general malaise and ongoing fatigue and an increased frequency in night terrors over the past few weeks. Her family attributes this to stress of school and her new job. She was noted to have lost $3 \mathrm{~kg}$ in the previous nine weeks.

\section{$\mathrm{PMH}, \mathrm{SH}$, and $\mathrm{FH}$}

Her PMH was unremarkable. She is a student and denies smoking, drinking or drug abuse. Her family history is noncontributory.

\section{Physical Examination}

- Vital signs: BP 100/60 mm Hg, P 90 beats/min and regular, R 16 breaths/min, T $100.8^{\circ} \mathrm{F}, \mathrm{BMI} 15$.

- Diffuse, non-tender lymphadenopathy through the submandibular and upper anterior cervical chains.

- Lungs: clear

- Heart: regular rhythm without murmur.

- Abdomen: slightly rounded and firm.

Which of the following are diagnostic considerations at this time?

1. Anorexia nervosa

2. Lymphoma

3. Mononucleosis

4. Teenage adjustment disorder

5. All of the above 


\section{Correct! \\ 5. All of the above}

The differential diagnosis in this clinical situation is quite broad. Teenage adjustment disorder is a stress-related condition that is short-term and occurs within 3 months of an event or situation. Teens with adjustment disorder experience depressed and irritable mood, sleep disturbances, and poor performance in school. The stress could be any adjustment from a loss, a breakup with a significant other, financial stress in the family, moving to a new town, to coping with divorce. Patients rarely have a BMI $<16$. A BMI of $<16$ in a teenager might suggest anorexia nervosa. Cervical lymph nodes can be prominent in teenagers but enlargement might suggest lymphoma and tuberculosis which can cause cervical lymphadenopathy and a low-grade fever.

Which of the following are not appropriate at this time?

1. Complete blood count

2. Chest x-ray

3. Mental health consultation

4. 1 and 3

5. All of the above 


\section{Correct! \\ 3. Mental health consultation}

Although the patient may have a mental health condition, there are some suggestions that she may have a physical ailment. Her low BMI and adenopathy could be due to anorexia but also could be secondary to a physical ailment such as infection (e.g., tuberculosis, histoplasmosis, mononucleosis), gastrointestinal disorders, cancer (e.g., lymphoma). For this reason, a CBC and chest $\mathrm{x}$-ray are indicated. The chest $\mathrm{x}$-ray is shown in Figure 1.

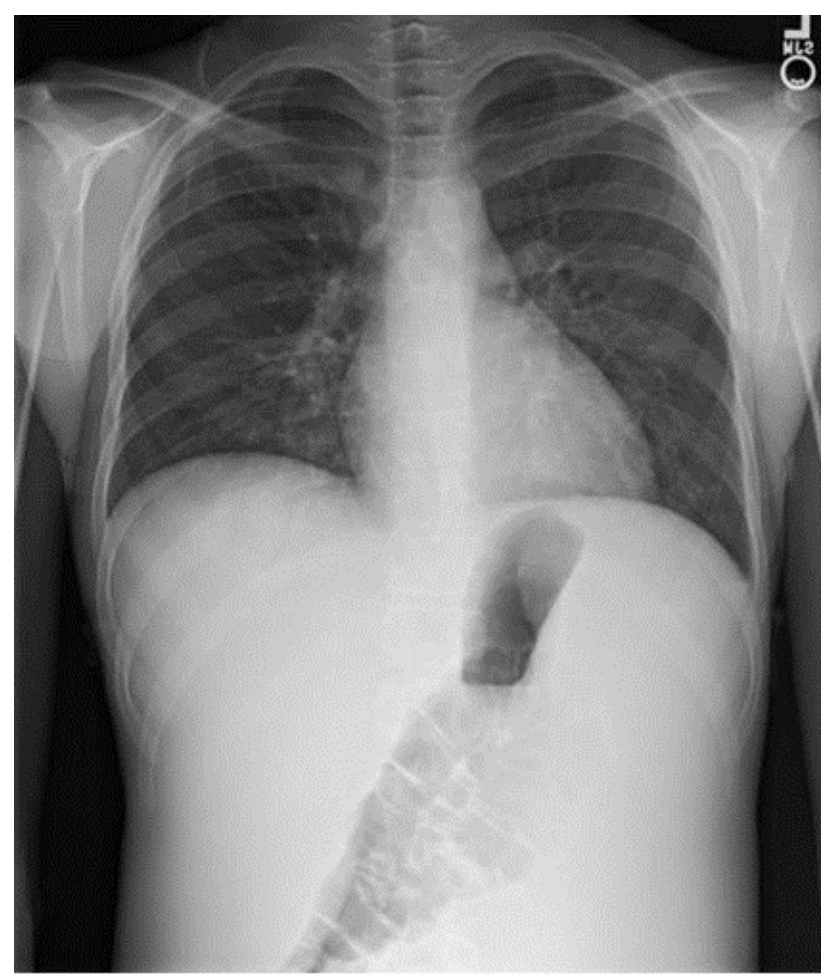

Figure 1. Initial PA of chest.

CBC showed a white blood cell count of greater than 400,000 and a platelet count of $1,357,000$. Hemoglobin was $7 \mathrm{~g} / \mathrm{dL}$. Additional laboratory obtained was a lactate dehydrogenase (LDH) which was elevated at $1980 \mathrm{IU} / \mathrm{L}$ (normal 110-283). Urinanalysis was unremarkable.

Which of the following is appropriate at this time?

1. Administration of IV fluids and allopurinol

2. Bone marrow biopsy

3. Hematology/oncology consultation

4. 1 and 3

5. All of the above 


\section{Correct! \\ 5. All of the above}

The chest x-ray is normal but the high white blood cell count is highly suggestive of leukemia. Therefore, hematology/oncology consultation is indicated. Bone marrow biopsy would be necessary for diagnosis and choosing the correct antineoplastic therapy. IV fluids and allopurinol are appropriate to prevent tumor lysis syndrome which likely with chemotherapy.

The patient was admitted to the hospital, blood cultures obtained, cephepime empirically ordered (to be discontinued if blood cultures are negative) and a bone marrow biopsy scheduled for the following morning. However, at 1:30 AM the rapid response team was called because of complaints of the sudden onset of shortness of breath, palpitations, and chest pain. Vital signs showed an Sp02 of 98\% with oxygen administered by blow, HR 133 beats/min, BP 105/63 mm Hg, RR 34 resp/min. Auscultation of the lung revealed bilateral crackles.

Which of the following are appropriate at this time?

1. Electrocardiogram (ECG)

2. IV furosemide for fluid overload

3. Thoracic CT angiogram

4. 1 and 3

5. All of the above 


\section{Correct!}

\section{1 and 3}

At this juncture it is not entirely clear the cause of her deteriation but clearly something dire has occurred. ECG is indicated as a screen for possible cardiac problems and the CT angiogram is indicated for possible pulmonary embolism. In the absence of a previous cardiac history, it would be unlikely that fluid overload would explain her sudden decline.

Her ECG is shown in Figure 2.

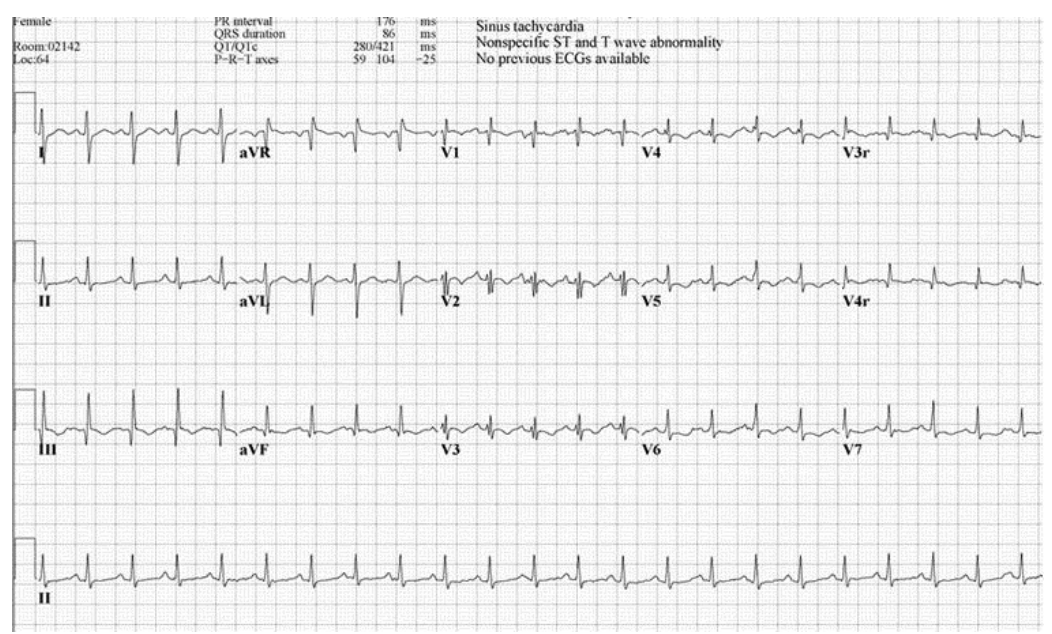

Figure 2. ECG.

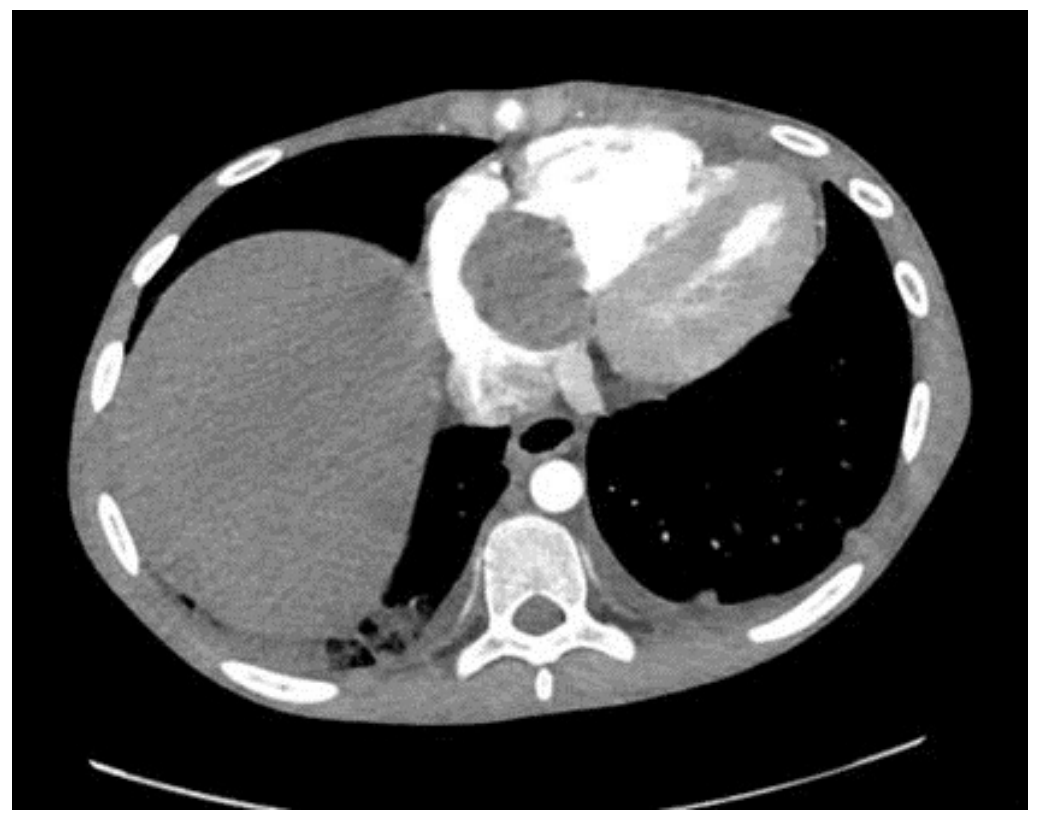

Figure 3. Representative image from thoracic CT angiogam. 
What is the cause of her shortness of breath, chest pain and palpitations?

1. Cardiac tamponade from pericardial effusion

2. Myocardial infarction (MI)

3. Pulmonary edema

4. Pulmonary embolism (PE)

5. None of the above 


\section{Correct! \\ 4. Pulmonary embolism}

The ECG shows an "S1Q3T3" pattern which is an S wave in lead 1 and a Q wave and inverted T wave in lead 3 . Although the ECG is usually considered a poor screening test for pulmonary embolism, the S1Q3T3 pattern is seen in about $50 \%$ of patients with massive PE (1). The thoracic CT angiogram confirms a massive PE with an intracardiac clot in the right atrium (Figure 4, red arrow).

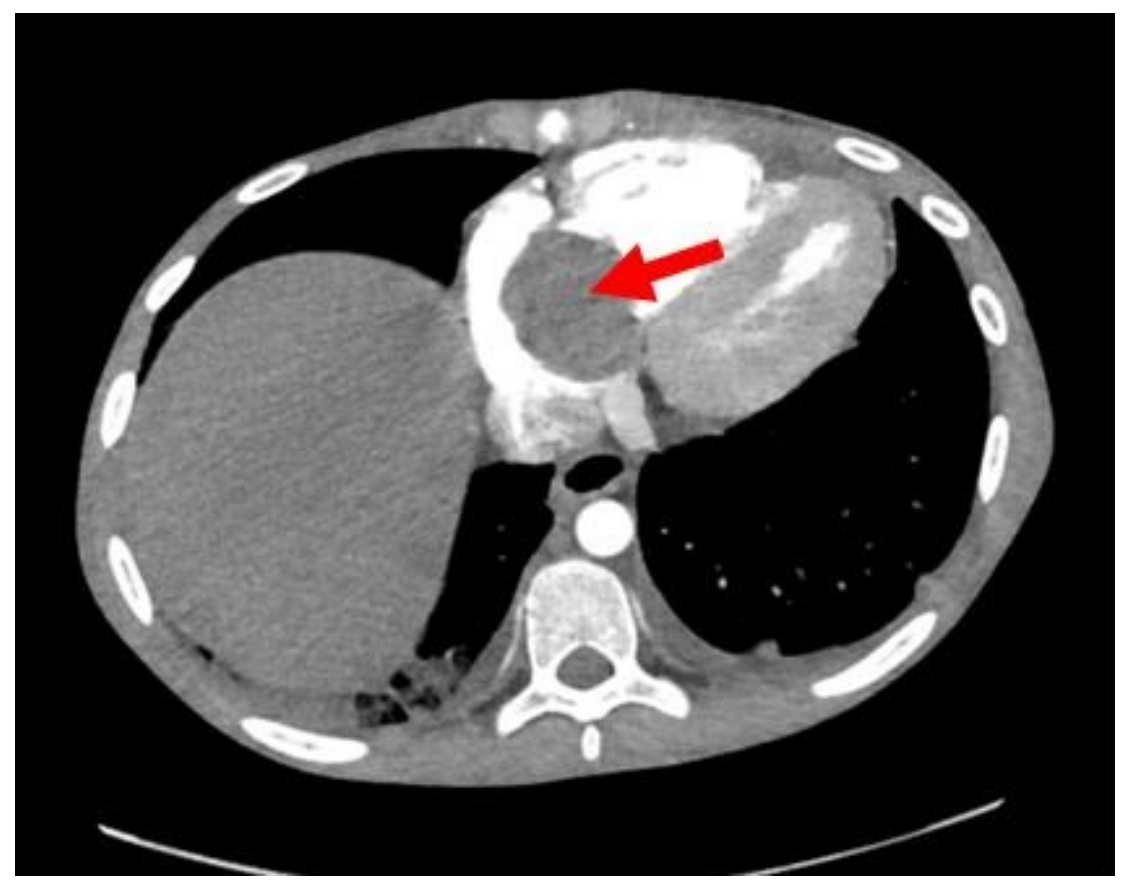

Figure 4. Thoracic CT angiogram showing the intracardiac clot (red arrow).

What therapy should the patient be given?

1. Catheter embolectomy

2. Heparin

3. Supportive care

4. Surgical embolectomy

5. Tissue plasminogen activator (TPA) followed by heparin 


\section{Correct! \\ 3. Supportive care}

Treatment in this situation relies heavily on clinical judgment and local expertise. It was thought that the patient was at too high a bleeding risk for anticoagulation or thrombolytic therapy. The patient was considered at too high a risk for surgical embolectomy. Although no controlled trials have been performed, catheter embolectomy is a consideration (2). The presence of the right atrial clot was judged to preclude catheter embolectomy. Therefore, supportive care is the best answer.

Shortly after returning to the pediatric intensive care unit from her CT angiogram, she became acutely anxious, asking for face mask oxygen. She then became agitated and combative. Her eyes rolled back, left eye deviated outwards. Her heart rate declined to the 40's with weak central pulses. Cardiopulmonary resuscitation (CPR) was initiated. She was endotracheally intubated and her pulse increased modestly although her oxygen saturation remained low at $70 \%$ with an inspired $\mathrm{FiO} 2$ of $100 \%$. A CT scan of the head without contrast revealed no apparent clot or hemorrhage. A repeat thoracic CT scan showed the atrial clot was smaller. A repeat echocardiogram revealed complete ventricular standstill with a severely dilated inferior vena cava. With agitated saline injection, there was no forward flow into the right ventricle nor through the right ventricular outflow tract.

Critically ill patients with an elevated platelet count are known to be at increased risk for pulmonary embolism (3). However, our patient was also at high bleeding risk. Some have advocated leukaphoresis early in the course of leukemia with high leukocyte counts. If respiratory and/or neurologic failure are present, the death rate at one week reaches $90 \%$ for patients with chronic mylogenous leukemia (CML). Leukapheresis reduces leukemic blast counts and associated symptoms until chemotherapy can be initiated (4). Whether either would have altered the patient's outcome is unclear.

\section{References}

1. Ferrari E, Imbert A, Chevalier T, Mihoubi A, Morand P, Baudouy M. The ECG in pulmonary embolism. Predictive value of negative $T$ waves in precordial leads--80 case reports. Chest. 1997 Mar;111(3):537-43. [CrossRef] [PubMed]

2. Kucher N. Catheter embolectomy for acute pulmonary embolism. Chest. 2007 Aug;132(2):657-63. [CrossRef] [PubMed]

3. Ho KM, Chavan S. Prevalence of thrombocytosis in critically ill patients and its association with symptomatic acute pulmonary embolism. A multicentre registry study. Thromb Haemost. 2013 Feb;109(2):272-9. [CrossRef] [PubMed]

4. Schiffer CA. Hyperleukocytosis and leukostasis in hematologic malignancies, UptoDate. 2016. Available at: http://www.uptodate.com/contents/hyperleukocytosisand-leukostasis-in-hematologicmalignancies?source=search result\&search=Hyperleukocytosis+and+leukostasis+in +hematologic+malignancies\&selectedTitle=1\%7E150 (accessed June 21, 2016, requires subscription). 